\title{
Off-axis multi-wavelength dispersion controlling metalens for multi-color imaging
}

\author{
Kaihua Dou ${ }^{1,2 \dagger}$, Xin Xie ${ }^{1,2+}$, Mingbo $\mathrm{Pu}^{1,2}$, Xiong $\mathrm{Li}^{1,2}$, Xiaoliang Ma ${ }^{1,2}$, \\ Changtao Wang ${ }^{1,2}$ and Xiangang Luo ${ }^{1,2 *}$
}

\begin{abstract}
Dispersion control is crucial in optical systems, and chromatic aberration is an important factor affecting imaging quality in imaging systems. Due to the inherent property of materials, dispersion engineering is complex and needs to trade off other aberration in traditional ways. Although metasurface offers an effective method to overcome these limits and results in well-engineered dispersion, off-axis dispersion control is still a challenging topic. In this paper, we design a single-layer metalens which is capable of focusing at three wavelengths $(473 \mathrm{~nm}, 532 \mathrm{~nm}$, and $632 \mathrm{~nm})$ with different incident angles $\left(0^{\circ},-17^{\circ}\right.$ and $\left.17^{\circ}\right)$ into the same point. We also demonstrate that this metalens can provide an alternative for the bulky color synthetic prism in a 3-chips digital micromirror device (DMD) laser projection system. Through this approach, various off-axis dispersion controlling optical devices could be realized.
\end{abstract}

Keywords: off-axis; dispersion control; metalens; color imaging

Dou K H, Xie X, Pu M B, Li X, Ma X L et al. Off-axis multi-wavelength dispersion controlling metalens for multi-color imaging. Opto-Electron Adv 3, 190005 (2020).

\section{Introduction}

Dispersion is an inherent property of a material. In general, dispersion would cause distortion of the signal and limit the operating bandwidth of devices. Dispersion control is an effective way to eliminate these effects. In general, dispersion control is mainly divided into two categories: amplification and elimination of dispersion. In spectroscopy and communication technology, strong dispersion is utilized to distinguish signals, such as spectrometer $^{1}$ and dense wavelength-division multiplexing $(\mathrm{DWDM})^{2}$. But in imaging systems, uncontrollable dispersion is a serious problem, which will greatly affect the imaging quality. In order to solve this problem, refraction and diffraction hybrid systems are generally used to eliminate the dispersion ${ }^{3}$. However, conventional refractive lenses are generally bulky and limited by the modulation mechanism, which will significantly constrain the performance of systems. With the development of processing technology and the increasingly high precision requirements, there is a potential to realize more compact, lightweight and integrated optical systems.

In recent years, as compact, lightweight devices which can break through the limitation of traditional optics, metasurfaces have attracted great attention of researchers ${ }^{4-6}$. As a subwavelength artificial electromagnetic material, the optical properties of metasurfaces depend on their materials and structural parameters. By optimizing the geometry size, arrangement and material properties of the sub-wavelength structure, one can flexibly control the amplitude, phase, and polarization of electromagnetic wave ${ }^{7,8}$. Such abilities show their functionalities in various applications, such as nonlinear frequency conversion $^{9}$, vortex beam generation ${ }^{10-12}$, holograms ${ }^{13-15}$, polarization manipulation ${ }^{16}$, flat lenses ${ }^{17-19}$, and virtual shaping $^{20-23}$, etc. Typically, metasurfaces can reduce the

\footnotetext{
${ }^{1}$ State Key Laboratory of Optical Technologies on Nano-Fabrication and Micro-Engineering, Institute of Optics and Electronics, Chinese Academy of Sciences, Chengdu 610209, China; ${ }^{2}$ School of Optoelectronics, University of Chinese Academy of Sciences, Beijing 100049 , China.

${ }^{\dagger}$ These authors contributed equally to this work.

*Correspondence: X G Luo, E-mail: Ixg@ioe.ac.cn

Received: 5 March 2019; Accepted: 7 April 2019; Published: 20 April 2020
} 
system redundancy, but the chromatic aberration still exists $^{24}$. Therefore, dispersion control has always been a key research topic in the design of metasurface devices. Previously reported works have studied a lot of the dispersion characteristics of metasurfaces. One method to control multi-wavelength dispersion is using the spatial multiplexing method ${ }^{18,25-27}$. In these designs, each phase shifter is optimized for one individual wavelength, and multi-wavelength modulation is realized by interleaving multiple phase shifters in a single pixel. Nevertheless, the interleaving and cross-talk between different structures for different wavelengths will degrade the imaging quality, and the energy utilization of the incident light cannot exceed $1 / n$, where $n$ is the number of modulation wavelengths. Recently, some dispersion-engineered approaches based on a single phase profile with wavelength dependence have been proposed to break the efficiency limitation ${ }^{17,28,29}$. Particularly, high refractive index and lossless titanium dioxide $\left(\mathrm{TiO}_{2}\right)$ is usually selected to design high-efficiency metasurface devices in visible regime $^{30,31}$. These metasurfaces are mainly applied for normal incidence. However, in practical applications, such as laser beams combination and laser projection display systems, dispersion control under oblique incidence is unavoidable. It should be noted that, although some pioneering works on off-axis dispersion control through metallic nano-groove gratings have been reported ${ }^{32}$, they still face the significant limitation of efficiency. Multi-wavelength and multi-incident angle operations can also be achieved by vertical stacking of metasurfaces ${ }^{33,34}$, while the volume and fabrication difficulty are inevitably increased.

In this work, we present a single-layer metalens to focus three lights with different wavelengths $(473 \mathrm{~nm}, 532$ $\mathrm{nm}$, and $632 \mathrm{~nm}$ ) into the same point at different incident angles $\left(0^{\circ},-17^{\circ}\right.$ and $\left.17^{\circ}\right)$. We first design the unit cells consisting of $\mathrm{TiO}_{2}$ nanopillars tiled on a dielectric spacer layer above a metallic mirror, which can provide special reflected phase responses at multi-wavelength. Then, to achieve the desired focus function, particle swarm optimization (PSO) algorithm is used to search the optimal phase profile and structure arrangement. Finally, we demonstrate that this metalens could provide an alternative for the color synthetic prism in a 3-chips DMD laser projection system, and the multi-color imaging behavior has been simulated.

\section{Principle and design}

To realize the focusing function, a phase retardation should be offered to change the incident plane wave to a converging wave. The phase retardation of the optical lens is required to compensate the phase difference of propagation in free space, the required phase $\varphi$ for incident wavelength $\lambda$ can be written as:

$$
\varphi\left(r, \lambda_{i}\right)=\frac{2 \pi\left(f-\sqrt{r^{2}+f^{2}}\right)}{\lambda_{i}}+\varphi_{\mathrm{d}}\left(\lambda_{i}\right)+C\left(\lambda_{i}\right), \quad i=1,2 \ldots n,
$$

where $f$ is the designed focal length, $r=\sqrt{x^{2}+y^{2}}$ indicates the distance from each pixel to the lens center, and $n$ is the number of operating wavelengths. In contrast to the previous achromatic metalenses which only consider the normal incidence conditions, here the additional phase $\varphi_{\mathrm{d}}$ $\left(\lambda_{i}\right)$ is used to compensate the off-axis (assuming the incident waves are in the $x z$-plane) aberration, as given by:

$$
\varphi_{\mathrm{d}}\left(\lambda_{i}\right)=\frac{2 \pi}{\lambda_{i}} x \sin \left(\theta_{i}\right), i=1,2 \ldots n,
$$

where $\theta$ represents the incident angle. The desired phase profile would focus the lights with different wavelengths and different incident angles to the same point, as depicted in Fig. 1(a). For practical application, the dispersion-engineered metalens could be used in multi-color
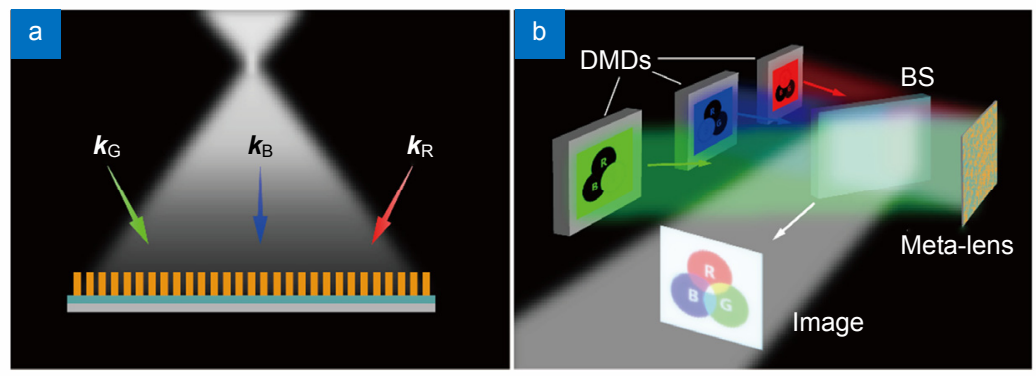

Fig. 1 | (a) Schematic of the off-axis multi-wavelength dispersion controlling metalens. The metasurface is illuminated by three lights with different wavelengths and incident angles, and then focuses the reflected lights into the same point. (b) Schematic of a 3-chips DMD laser projection system with the dispersion-engineered metalens. The three monochromatic $(R, G$ and $B)$ images can be synthesized into a color image after reflected by the metasurface. BS: beam splitter. 


\section{Opto-Electronic Advances https://doi.org/10.29026/oea.2020.190005}

laser projection systems. Specifically, as shown in Fig. $1(b)$, three monochromatic red $(R)$, green $(G)$, and blue (B) projections from DMDs are reflected by the metasurface and finally form the output colorful images in the focal plane. According to the working principle of $\mathrm{DMD}^{35}$, the specific incident angles for the metalens are set as $0^{\circ},-17^{\circ}$ and $17^{\circ}$ for three lights, respectively. The focusing function is determined by the first two parts in equation (1), $C\left(\lambda_{i}\right)$ is a constant phase and acts as a tuning knob in the design. We find the optimal value of $C\left(\lambda_{i}\right)$ using the PSO algorithm to match the desired phase profile. Hence, we design a metalens with the numerical aperture (NA) of 0.38 and a focal length of $50 \mu \mathrm{m}$ (Fig. 2(a)). Three lights with wavelengths of $473 \mathrm{~nm}, 532 \mathrm{~nm}, 632$ $\mathrm{nm}$ irradiate on the metasurface at incident angles of $0^{\circ}$, $-17^{\circ}$ and $17^{\circ}$, respectively, and finally focus into the same point.

From equations (1) and (2), the phase function varies with the wavelength and incident angle, which is the inherent dispersion in the process of light propagation. General achromatic metalenses just need to compensate the regular phase retardation of propagation in free space. However, in our case, the off-axis incident lights need to be deflected, thus the phase profile of each wavelength is no longer central symmetry distribution. The phase concentric rings deviate along the incident direction. We denote $\varphi_{\lambda}(x, y)$ as the desired phase of one pixel at coordinate $(x, y)$ for the wavelength $\lambda$. Here, the required phases at a pixel for different wavelengths may have a great difference (i.e., $\left.\varphi_{\lambda_{1}}\left(x_{1}, y_{1}\right) \neq \varphi_{\lambda_{2}}\left(x_{1}, y_{1}\right)\right)$. Besides, the phases at different pixels are equal for one wavelength, but different for another wavelength (i.e., $\varphi_{\lambda_{1}}\left(x_{1}, y_{1}\right)=$ $\left.\varphi_{\lambda 1}\left(x_{2}, y_{2}\right), \varphi_{\lambda 2}\left(x_{1}, y_{1}\right) \neq \varphi_{\lambda 2}\left(x_{2}, y_{2}\right)\right)$. We design a single phase profile with wavelength dependence to modulate the three reflected waves (Fig. 2(a)). To achieve the desired function, we first build a structure-phase library which contains large phase response range. Parametric sweep is performed with a uniform step to obtain the corresponding reflected phase. Then we select the suitable structure from the library using PSO algorithm to match the desired phase at each pixel.

To suppress the energy loss caused by high-order diffraction, the period of unit cells is selected to satisfy the Nyquist sampling criterion and should be less than $\lambda / 2 \mathrm{NA}$. As shown in Fig. 2(b), we design a unit cell with a period of $400 \mathrm{~nm}$. A square $\mathrm{TiO}_{2}$ nanopillar with a height
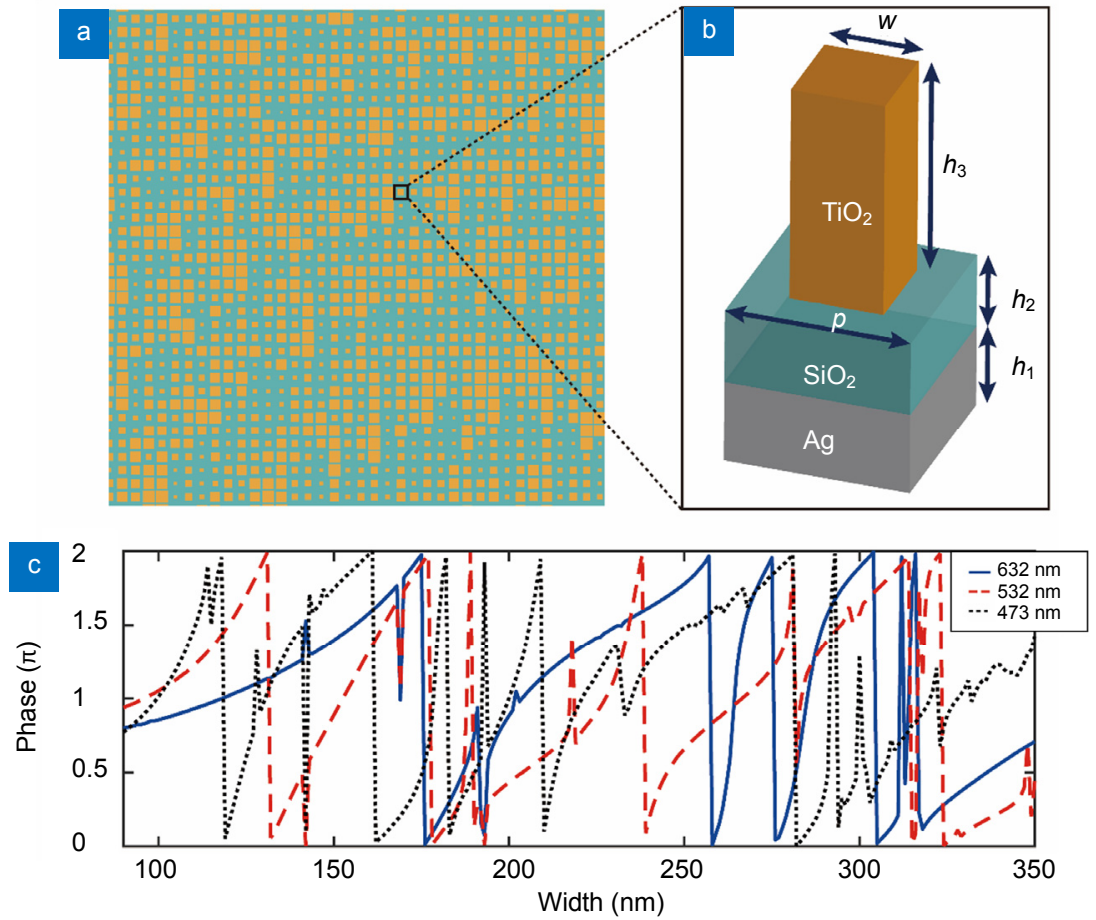

Fig. 2 | (a) Top-view of part of the designed metalens. (b) The element consists of a titanium dioxide $\left(\mathrm{TiO}_{2}\right)$ nanopillar $\left(h_{3}=530 \mathrm{~nm}\right)$ on top of a silicon dioxide $\left(\mathrm{SiO}_{2}\right)$ layer $\left(h_{2}=180 \mathrm{~nm}\right)$ above a silver substrate $\left(h_{1}=200 \mathrm{~nm}\right)$. The period $p=400 \mathrm{~nm}$, the nanopillar has a square cross-section with width $w$. By adjusting the width, the reflection phase can be controlled. (c) Numerically calculated reflection phase shift as a function of $w$ at three wavelengths of $473 \mathrm{~nm}, 532 \mathrm{~nm}$, and $632 \mathrm{~nm}$. As the nanopillar width $w$ changes from $90 \mathrm{~nm}$ to $350 \mathrm{~nm}$, the corresponding phase undergoes multiple $0-2 \pi$ phase cycles. The refractive indexes of $\mathrm{TiO}_{2}$ are $2.493,2.43$ and 2.373 for $473 \mathrm{~nm}, 532 \mathrm{~nm}$, and $632 \mathrm{~nm}$ in the simulations, respectively. 
of $530 \mathrm{~nm}$ is placed on a $180 \mathrm{~nm}$ thick $\mathrm{SiO}_{2}$ layer and a $200 \mathrm{~nm}$ thick silver is used as a reflective layer at the bottom. This subwavelength structure functions as a waveguide and supports guided mode resonances ${ }^{36,37}$. Abnormal phase response occurs to decouple the realized phases at different wavelengths ${ }^{29}$. Using the commercial software, Computer Simulation Technology (CST) Microwave Studio, we sweep the width of the nanopillar (ranging from $90 \mathrm{~nm}$ to $350 \mathrm{~nm}$ ) to obtain reflected phase response. Then we use these geometry parameters and the corresponding phase responses to build the structure-phase library. The simulated phase response is folded into multiple $2 \pi$ cycles, as shown in Fig. 2(c). For the short wavelength of $473 \mathrm{~nm}$ which is close to the period, stronger guided mode resonance would be excited, resulting in large phase coverage and more phase jumps in the same width range. While for the long wavelength of $632 \mathrm{~nm}$, the resonance becomes quite weak. Taking advantage of this anomalous dispersion property, we can use the single phase shifter to modulate the local phase for three wavelengths.

\section{Results and discussion}

Rayleigh criterion holds that the wavefront can be regarded as perfect when the maximum wave aberration between the actual wavefront and the ideal wavefront does not exceed $\lambda / 4$. Moreover, if the defect portion occupies a small proportion of the total wavefront product, these local defects can be ignored even if the wave phase difference is greater than $\lambda / 4$. This means that we do not need to find a subwavelength structure fully meets the ideal phase requirements, just need to meet most of the phase response requirements. To verify the performance of the off-axis multi-wavelength dispersion controlling metalens, a full model with the diameter of $40.4 \mu \mathrm{m}$ and a focal length of $50 \mu \mathrm{m}$ was designed (Fig. 2(a)). We discretize the phase profile into a matrix containing $101 \times 101$ pixels, and the ideal phase can be obtained by equations (1). In order to search the structure which is most suitable for the ideal phase, we need to find the structure phase response $\varphi_{\mathrm{s}}$ closest to the desired phase $\varphi_{\mathrm{d}}$. Here, we perform this process by searching the minimum value of $\Delta \varphi=\varphi_{\mathrm{d}}-\varphi_{\mathrm{s}}$ utilizing the PSO method. The ideal phase and optimized phase distributions for different wavelengths can be seen in Fig. 3. We calculate the phase error at each pixel on the metalens, which is the difference between the target phase (Fig. 3(a)) and actual phase (Fig. $3(\mathrm{~b})$ ), as seen in Fig. S1(a). The mean absolute errors of phase profile are $17.1^{\circ}, 23.5^{\circ}$ and $22.9^{\circ}$ for the three wavelengths, respectively, the error histogram for each wavelength is shown in Fig S2(a). The corresponding reflectivity of each pixel is also calculated as shown in Figs. S1(b) and S2(b). The simulated focusing efficiencies are $47.82 \%, 52.04 \%$ and $51.82 \%$ for $473 \mathrm{~nm}, 532 \mathrm{~nm}$, and $632 \mathrm{~nm}$, respectively.

To evaluate the performance of the optimized phase distribution, we first calculate the intensity profile in the focal plane by vector angular spectrum theory. Then we obtain the corresponding structure parameters according to optimized phase distribution. Subsequently, full-wave simulations are carried out using CST Microwave Studio. In order to obtain the far-field intensity distribution at the

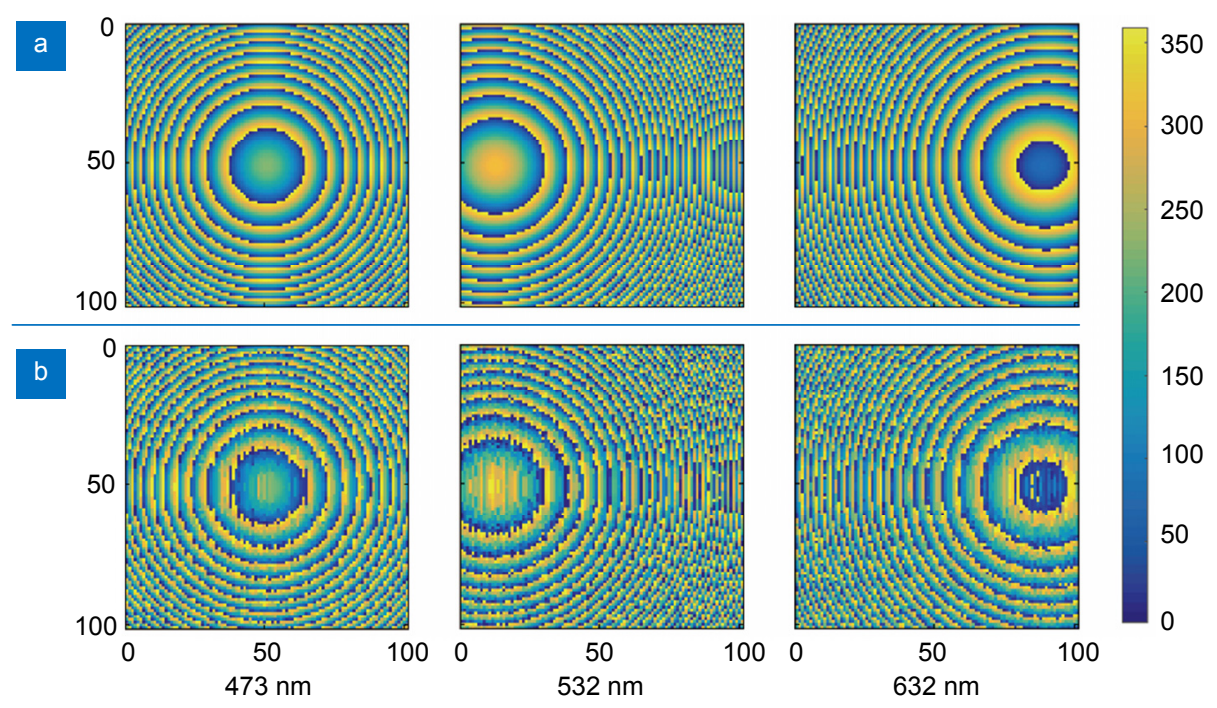

Fig. 3 | Comparison between the target phase profiles (a) and optimized results (b) at three wavelengths of $473 \mathrm{~nm}$ (left), $532 \mathrm{~nm}$ (middle), and $632 \mathrm{~nm}$ (right). The designed metasurface consists of 101×101 pixels. Abscissa and ordinate indicate the number of pixels. 


\section{Opto-Electronic Advances https://doi.org/10.29026/oea.2020.190005}

focal plane, we calculate Fraunhofer diffraction of the surface field obtained from CST. Owing to the geometrical symmetry of the square pillar, the metasurface is insensitive to the polarization states of the incoming lights when the incident angle is small. The calculated results under transverse electric (TE) polarized illumination are given in Figs. 4(a)-4(c), which show normalized intensity distributions in the $y z$-plane for three wavelengths of $473 \mathrm{~nm}, 532 \mathrm{~nm}$, and $632 \mathrm{~nm}$. The corresponding focal lengths are respectively $49.9 \mu \mathrm{m}, 49.45 \mu \mathrm{m}$ and $50.05 \mu \mathrm{m}$. Figs. 4(d) $-4(\mathrm{f})$ illustrate the calculated normalized intensity profiles of the three lights along the $y$-axis across the focal plane, the full-widths at half maximums (FWHMs) are $592 \mathrm{~nm}, 669 \mathrm{~nm}$, and $730 \mathrm{~nm}$, respectively, agree well with the theoretical counterparts $(622 \mathrm{~nm}, 700 \mathrm{~nm}$, and $831 \mathrm{~nm}$, respectively). The slight deviations may be the result of approximate local phase distribution and a limited number of pixels in the simulations. The normalized intensity distributions in the $x y$ plane at the focal plane for three wavelengths of $473 \mathrm{~nm}$, $532 \mathrm{~nm}$, and $632 \mathrm{~nm}$ are also provided as shown in Figs. 4(g)-4(i). To demonstrate the polarization-independent property, the results for transverse magnetic (TM) polarized incidence are presented in Fig. S3, which show good agreement with that of TE polarized one. In general, the metalens exhibits excellent performance in off-axis multi-wavelength dispersion control.

In the following, we demonstrate the multi-color imaging of our metalens. First, we decompose a target full-color image into its RGB components. Then three DMD chips are used to generate monochromatic images as the incident sources, three monochromatic (R-632 nm, G-532 nm, and B-473 nm) images would be focused onto the center in the focal plane after reflected by the
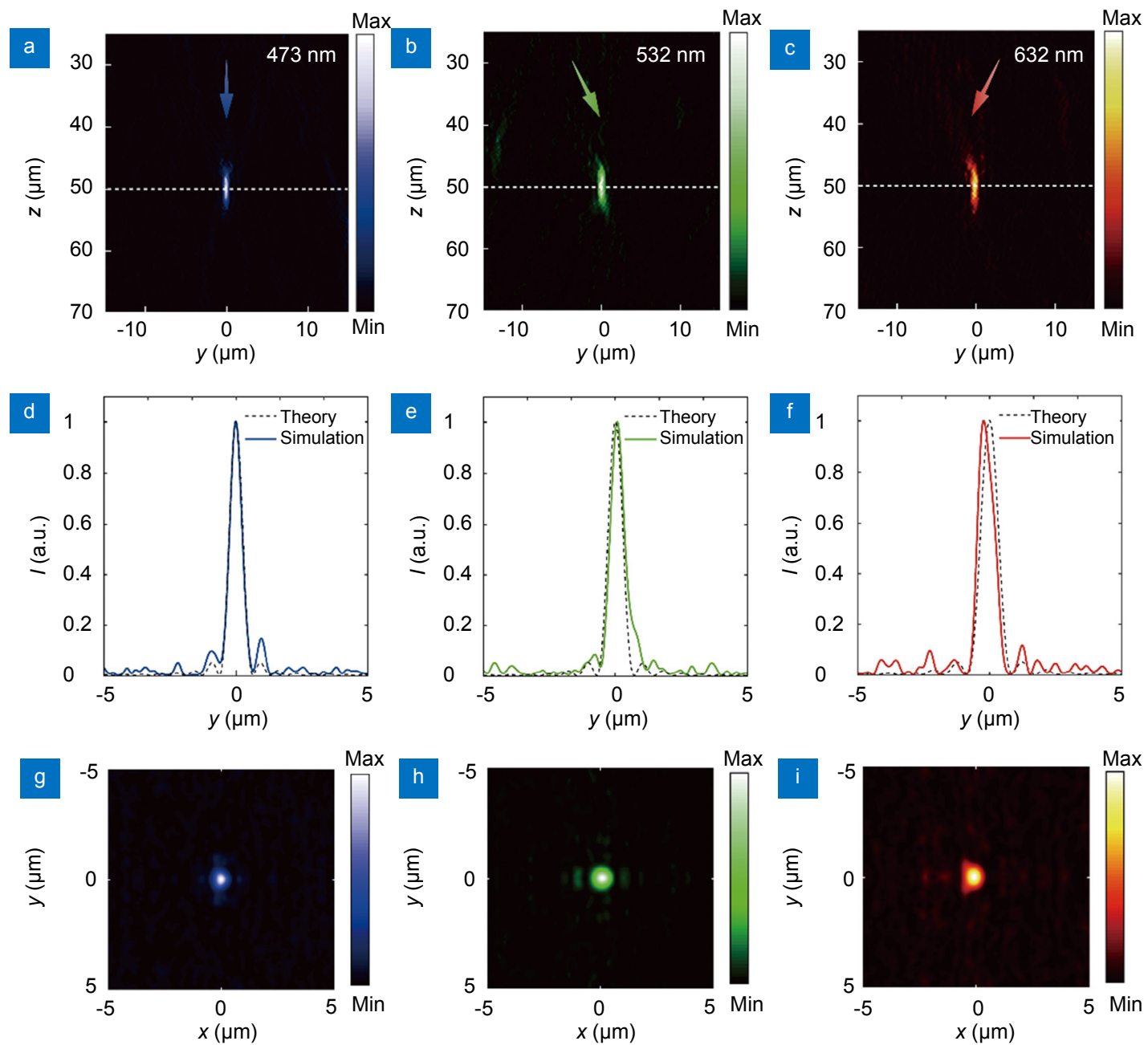

Fig. 4 | (a-c) Numerically calculated normalized intensity distributions in the $y z$-plane at the wavelengths of $473 \mathrm{~nm}, 532 \mathrm{~nm}$, and $632 \mathrm{~nm}$ under TE polarized incidence. The corresponding incident angles are $0^{\circ},-17^{\circ}$ and $17^{\circ}$, respectively. (d-f) Theoretical (dotted lines) and calculated (solid lines) normalized intensity curves of the three lights along the $y$-axis across the focal plane. (g-i) Normalized intensity distributions in the $x y$-plane at the focal plane for the wavelengths of $473 \mathrm{~nm}, 532 \mathrm{~nm}$, and $632 \mathrm{~nm}$. 
metalens, as shown in Figs. 5(a)-5(c). Owing to the ununiformed efficiency of the metalens for the three wavelengths, the reflected R, G and B images have an intensity ratio of 1: 0.878: 0.667 for the incidences with the same power. In order to make the synthetic color match with the target, we adjust the intensities of incident R, G, and B lights in the ratio of $1: 1.138$ (i.e. 1/0.878): 1.5 (i.e. 1/0.667). For the three monochromatic images illuminating on the metalens simultaneously, the output signal will be a synthetic color image, as shown in Fig. 5(d).
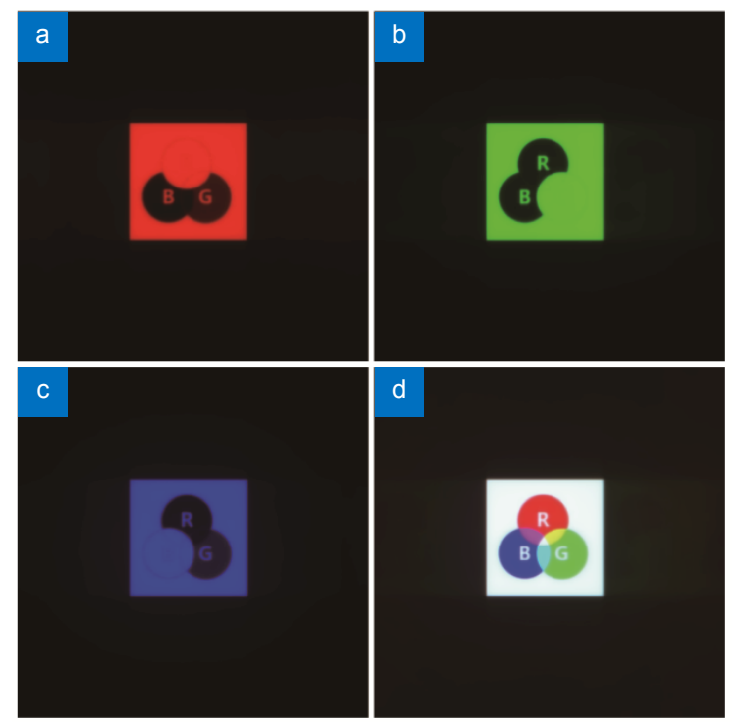

Fig. 5 | (a-c) Simulated monochromatic imaging by the metalens for three wavelengths of $473 \mathrm{~nm}, 532 \mathrm{~nm}$, and $632 \mathrm{~nm}$ with incident angles of $0^{\circ},-17^{\circ}$ and $17^{\circ}$, respectively. (d) Synthetic color image by the metalens.

\section{Conclusion}

In summary, we have demonstrated a single-layer metasurface that can realize off-axis $\left( \pm 17^{\circ}\right)$ multi-wavelength ( $473 \mathrm{~nm}, 532 \mathrm{~nm}$, and $632 \mathrm{~nm}$ ) dispersion engineering in the visible spectrum. Multi-color laser lensing and imaging are well demonstrated. Along with the design of metasurface with free phase for each wavelength, this design can break the constraints of conventional optics and explore new components with desired dispersion. We believe that this work could offer a new access for the design of off-axis multi-wavelength dispersion-engineered metadevices, and may find promising applications in multi-color imaging optical systems.

\section{References}

1. Yang C A, Edwards P, Shi K B, Liu Z W. Proposal and demonstration of a spectrometer using a diffractive optical element with dual dispersion and focusing functionality. Opt Lett 36, 2023-2025 (2011).
2. Gong $Y D, L i T$ J, Jian $S$ S. Multi-channel fiber grating for DWDM. Chin J Electron 9, 292-295 (2000).

3. Stone T, George N. Hybrid diffractive-refractive lenses and achromats. Appl Opt 27, 2960-2971 (1988).

4. Luo X G, Tsai D, Gu M, Hong M H. Extraordinary optical fields in nanostructures: from sub-diffraction-limited optics to sensing and energy conversion. Chem Soc Rev 48, 2458-2494 (2019).

5. Luo X G, Tsai D, Gu M, Hong M H. Subwavelength interference of light on structured surfaces. Adv Opt Photonics 10, 757-842 (2018).

6. Nemati $A$, Wang $Q$, Hong $M \mathrm{H}$, Teng $\mathrm{J} \mathrm{H}$. Tunable and reconfigurable metasurfaces and metadevices. Opto-Electron Adv 1, 180009 (2018).

7. Luo X G. Subwavelength artificial structures: opening a new era for engineering optics. Adv Mater 31, 1804680 (2019).

8. Yu N F, Capasso F. Flat optics with designer metasurfaces. Nat Mater 13, 139-150 (2014).

9. Rahmani M, Leo G, Brener I, Zayats A V, Maier S A et al. Nonlinear frequency conversion in optical nanoantennas and metasurfaces: materials evolution and fabrication. Opto-Electron Adv 1, 180021 (2018).

10. Guo $Y$ H, Pu M B, Zhao Z Y, Wang Y Q, Jin J J et al. Merging geometric phase and plasmon retardation phase in continuously shaped metasurfaces for arbitrary orbital angular momentum generation. ACS Photonics 3, 2022-2029 (2016).

11. Jin J J, Pu M B, Wang Y Q, Li X, Ma X L et al. Multi-channel vortex beam generation by simultaneous amplitude and phase modulation with two-dimensional metamaterial. Adv Mater Technol 2, 1600201 (2017).

12. Pu M B, Li X, Ma X L, Wang Y Q, Zhao Z Y et al. Catenary optics for achromatic generation of perfect optical angular momentum. Sci Adv 1, e1500396 (2015).

13. Li X, Chen L W, Li Y, Zhang X H, Pu M B et al. Multicolor 3D meta-holography by broadband plasmonic modulation. Sci Adv 2, e1601102 (2016).

14. Almeida E, Bitton O, Prior Y. Nonlinear metamaterials for holography. Nat Commun 7, 12533 (2016).

15. Zheng G X, Mühlenbernd H, Kenney M, Li G X, Zentgraf T et al. Metasurface holograms reaching $80 \%$ efficiency. Nat Nanotechnol 10, 308-312 (2015).

16. Ma X L, Pu M B, Li X, Guo Y H, Luo X G. All - metallic wide angle metasurfaces for multifunctional polarization manipulation. Opto - Electron Adv 2, 180023 (2019).

17. Khorasaninejad M, Shi Z, Zhu A Y, Chen W T, Sanjeev V et al. Achromatic metalens over $60 \mathrm{~nm}$ bandwidth in the visible and metalens with reverse chromatic dispersion. Nano Lett 17, 1819-1824 (2017).

18. Chen B H, Wu P C, Su V C, Lai Y C, Chu C H et al. GaN metalens for pixel-level full-color routing at visible light. Nano Lett 17, 6345-6352 (2017).

19. Pu M B, Li X, Guo Y H, Ma X L, Luo X G. Nanoapertures with ordered rotations: symmetry transformation and wide-angle flat lensing. Opt Express 25, 31471-31477 (2017).

20. Ni X J, Wong Z J, Mrejen M, Wang Y, Zhang X. An ultrathin invisibility skin cloak for visible light. Science 349, 1310-1314 (2015).

21. Pu M B, Zhao Z Y, Wang Y Q, Li X, Ma X L et al. Spatially and spectrally engineered spin-orbit interaction for achromatic virtual shaping. Sci Rep 5, 9822 (2015).

22. Xie X, Li X, Pu M B, Ma X L, Liu K P et al. Plasmonic metasurfaces for simultaneous thermal infrared invisibility and 
holographic illusion. Adv Funct Mater 28, 1706673 (2018).

23. Xie $X$, Pu M B, Huang $Y J$, Ma X L, Li $X$ et al. Heat resisting metallic meta-skin for simultaneous microwave broadband scattering and infrared invisibility based on catenary optical field. Adv Mater Technol 4, 1800612 (2019).

24. Ozaki M, Kato J I, Kawata S. Surface-plasmon holography with white-light illumination. Science 332, 218-220 (2011).

25. Li K, Guo Y H, Pu M B, Li X, Ma X L et al. Dispersion controlling meta-lens at visible frequency. Opt Express 25, 21419-21427 (2017).

26. Lin D M, Holsteen A L, Maguid E, Wetzstein G, Kik P G et al. Photonic multitasking interleaved si nanoantenna phased array. Nano Lett 16, 7671-7676 (2016).

27. Arbabi E, Arbabi A, Kamali S M, Horie Y, Faraon A. Multiwavelength polarization-insensitive lenses based on dielectric metasurfaces with meta-molecules. Optica 3, 628-633 (2016).

28. Khorasaninejad M, Chen W T, Oh J, Capasso F. Super-dispersive off-axis meta-lenses for compact high resolution spectroscopy. Nano Lett 16, 3732-3737 (2016).

29. Shi $Z$ J, Khorasaninejad M, Huang Y W, Roques-Carmes C, Zhu A $Y$ et al. Single-layer metasurface with controllable multiwavelength functions. Nano Lett 18, 2420-2427 (2018).

30. Fan Q B, Zhu W Q, Liang Y Z, Huo P C, Zhang C et al. Broadband generation of photonic spin-controlled arbitrary accelerating light beams in the visible. Nano Lett 19, 1158-1165 (2019).

31. Fan $Q B$, Huo $P$ C, Wang D P, Liang $Y Z$, Yan $F$ et al. Visible light focusing flat lenses based on hybrid dielectric-metal metasurface reflector-arrays. Sci Rep 7, 45044 (2017).

32. Deng $Z \mathrm{~L}$, Zhang $S$, Wang G P. Wide-angled off-axis achromatic metasurfaces for visible light. Opt Express 24, 23118-23128
(2016).

33. Liu $S$, Zhang L, Yang $Q L$, Xu Q, Yang $Y$ et al. Frequency-dependent dual-functional coding metasurfaces at terahertz frequencies. Adv Opt Mater 4, 1965-1973 (2016).

34. Arbabi A, Arbabi E, Kamali S M, Horie Y, Han S et al. Miniature optical planar camera based on a wide-angle metasurface doublet corrected for monochromatic aberrations. Nat Commun $\mathbf{7}$, 13682 (2016).

35. The working principle of DMD. (last accessed December 3 , 2018); http://www.ti.com.cn/product/cn/dlp480re/

36. Wang S S, Magnusson R. Theory and applications of guided-mode resonance filters. Appl Opt 32, 2606-2613 (1993).

37. Fan $\mathrm{S} \mathrm{H}$, Joannopoulos $\mathrm{J} \mathrm{D}$. Analysis of guided resonances in photonic crystal slabs. Phys Rev B 65, 235112 (2002).

\section{Acknowledgements}

We acknowledge the financial support by the National Natural Science Foundation of China under contact Nos. 61622508, 61622509, and 61575201.

\section{Competing interests}

The authors declare no competing financial interests.

\section{Supplementary information}

Supplementary information for this paper is available at https://doi.org/10.29026/oea.2020.190005 